\title{
Efeito da massagem miofascial sobre a dor e a propriocepção pós-mastectomia radical
}

\section{Effect of miofascial massage on pain on proprioception of women after modified radical mastectomy}

\author{
Débora Sanfelice Zanon, Ana Carla Piovesan, Melissa Medeiros Braz, Sara \\ Teresinha Corazza, Hedioneia Maria Foletto Pivetta
}

http://dx.doi.org/10.11606/issn.2238-6149.v28i1p115-121

Zanon DS, Piovesan AC, Braz MM, Corazza ST, Pivetta HMF. Efeito da massagem miofascial sobre a dor e a propriocepção pós-mastectomia radical. Rev Ter Ocup Univ São Paulo. 2017 jan.-abr.;28(1):115-21.

RESUMO: Introdução: o câncer de mama, dentre as neoplasias malignas, tem sido o responsável pelos maiores índices de mortalidade no mundo. Diversos problemas podem estar relacionados à cirurgia, como a dor e as alterações na sensibilidade, os quais são responsáveis por uma maior tensão miofascial, que leva à diminuição na amplitude dos movimentos, estando diretamente relacionados à propriocepção. Objetivos: analisar o efeito agudo da abordagem indireta no tecido conjuntivo sobre a dor e a propriocepção de mulheres submetidas à mastectomia radical modificada. Método: a pesquisa é do tipo quase experimental com pré e pós-teste, composta por mulheres com idades entre 40 e 72 anos. Foram realizadas as seguintes avaliações: questionário sociodemográfico; questionário Disabilities of the Arm, Shoulder and Hand; Escala Analógica da dor e cinesiômetro para a avaliação da propriocepção. Após, as mulheres foram submetidas à aplicação da massagem miofascial. Para a análise dos dados, foi realizado o teste de normalidade de Shapiro-Wilk, sendo que nos dados simétricos, foi utilizado o Teste $\mathrm{T}$ Pareado, e nos dados assimétricos, foi utilizado o teste Wilcoxon. Resultados: participaram da pesquisa 12 mulheres. Os resultados demonstraram que houve redução nas médias, o qual, estatisticamente, não foram significativos em relação à dor e à propriocepção, pré e pós-abordagem indireta do tecido conjuntivo. Conclusão: concluiu-se que não houve melhora da dor e propriocepção após massagem miofascial em mulheres mastectomizadas.

DESCRITORES: Mastectomia radical; Propriocepção; Saúde da mulher; Massagem.
Zanon DS, Piovesan AC, Braz MM, Corazza ST, Pivetta HMF. Effect of miofascial massage on pain on proprioception of women after modified radical mastectomy. Rev Ter Ocup Univ São Paulo. 2017 Jan.-Apr.;28(1):115-21.

ABSTRACT Introduction: breast cancer, among malignant neoplasms, has been responsible for the highest mortality rates in the world. Several problems can be related to the surgery, such as pain and changes in sensitivity, which are responsible for a higher myofascial strain that reduces the amplitude of movements - being directly related to proprioception. Objectives: to analyze the acute effect of an indirect management of the connective tissue on the pain and proprioception of women subjected to modified radical mastectomy. Method: this research is of the quasiexperimental type, with pre- and post-test, with a sample composed of women aged between 40 and 72 years. The following assessments were performed: socio-demographic questionnaire; Disabilities of the Arm, Shoulder and Hand (DASH) questionnaire; pain analog scale and a kinesimeter for the proprioception assessment. After this, the women underwent myofascial massage. In the data analysis, we conducted the Shapiro-Wilk test for normality. For symmetric data, we used paired T-test, and the Wilcoxon for asymmetric data. Results: 12 women participated in the research. The results showed a reduction in the averages, which were not statistically significant in relation to pain and proprioception, and to indirect pre- and postmanagement of the connective tissue. Conclusion: we found no improvement in pain and proprioception after myofascial massage in mastectomized women.

KEYWORDS: Mastectomy radical; Proprioception; Women's health; Massage.

Artigo resultante da Monografia de Especialização do Curso de Reabilitação Físico-Motora da Universidade Federal de Santa Maria.

1. Universidade Federal de Santa Maria.E-mails: debora.s.z.2012@hotmail.com, aninhapiovesan@yahoo.com.br, melissabraz@hotmail.com, saratcorazza@gmail.com,hedioneia@yahoo.com.br

Endereço para correspondência: Hedioneia Maria Foletto Pivetta. Av. Roraima, 1000. Cidade Universitária. Bairro Camobi. Santa Maria, RS. CEP: 97.105-900. 


\section{INTRODUÇÃO}

A incidência de câncer vem aumentando, cada vez mais, convertendo-se em um evidente problema de saúde pública mundial. A Organização Mundial da Saúde (OMS) ${ }^{1}$ estimou que, para o ano 2030, podem ser esperados 27 milhões de casos de câncer no mundo.

O câncer é definido como uma doença causada por exacerbadas e incontroláveis divisões de células anormais que têm alterações morfológicas e funcionais, com capacidade de invadir tecidos e estruturas regionais a distância, o que pode levar o indivíduo à morte ${ }^{2}$. O câncer de mama é o tumor mais comum entre mulheres, com alta taxa de incidência e mortalidade ${ }^{3}$.

Como abordagem terapêutica, a cirurgia consiste na principal escolha, tendo em vista a possibilidade de erradicar o tumor. A mastectomia radical modificada é o tipo mais comum de mastectomia, em que ocorre a retirada total da mama, com o esvaziamento axilar e pode ser classificada como mastectomia "a Patey", quando o músculo peitoral maior é preservado, ou "a Madden", quando ocorre a preservação de ambos os músculos peitorais ${ }^{4}$.

Este tipo de tratamento está associado ao surgimento de diversas alterações funcionais, sequelas e complicações como, por exemplo, quadro postural assimétrico; alterações vasculares e linfáticas; aderências na parede torácica; alinhamento anormal das escápulas, como resultado de uma mudança súbita no peso corporal, no hemicorpo cirurgiado e, ainda, limitação do movimento do ombro; alterações de sensibilidade nas regiões do braço e axila e retração miofascial ${ }^{5}$.

Uma das complicações que podem ocorrer com a mulher é a alteração da propriocepção. Entende-se esta como a percepção do segmento no espaço, bem como a posição, velocidade, distância e direção do movimento a ser realizado. Sendo assim, a propriocepção é a capacidade em reconhecer a localização espacial do corpo, sua posição e orientação, a força exercida pelos músculos e a posição de cada parte do corpo em relação às demais, sem que seja utilizada a visão $0^{6,7}$.

Os proprioceptores, por sua vez, são estruturas presentes nas articulações, nos músculos, nos ligamentos e tendões. São estruturas responsáveis por enviar ao Sistema Nervoso Central todo o tipo de informação relacionada à organização espacial dos segmentos, ou seja, sobre as relações biomecânicas dos tecidos articulares, as quais podem influenciar no tônus muscular, no equilíbrio postural e na estabilidade $\operatorname{articular}^{6,7}$.
Dessa forma, pode-se presumir que a capacidade de interpretação das informações advindas do membro superior sofre influências diretas da tensão miofascial, resultantes dos procedimentos no hemitórax acometido pelo câncer de mama, o que resulta na diminuição da qualidade do movimento, pois este está diretamente relacionado à propriocepção.

A miofáscia é um tipo específico de fáscia profunda ligada ao tecido muscular. A massagem miofascial enfoca a unidade miofascial, incluindo o músculo, o tecido conjuntivo e a junção neuromuscular. A massagem miofascial é um recurso da terapia manual de baixo custo e ampla efetividade para alterações neuromusculoesqueléticas. Dentre as modalidades dessa técnica, a abordagem indireta do tecido conjuntivo usa apenas movimentos suaves para a liberação da miofáscia, útil para tratar áreas com dor e inflamação aguda ${ }^{8}$.

Os padrões de compensação postural e diminuição da elasticidade (diminuição da amplitude de movimento por encurtamento muscular), associados a linhas dos trilhos anatômicos do membro superior levam a variados tipos de disfunções envolvendo ombro, braço e mão, e são comumente relacionados ao fato de os ombros estarem protraídos, retraídos, elevados ou rodados internamente ${ }^{9}$. No caso de mulheres mastectomizadas, alterações funcionais importantes surgem no membro superior do lado cirurgiado, principalmente, no que tange à restrição de amplitude de movimento, dor e alteração de sensibilidade.

Frente a essas manifestações, a reabilitação assume um papel fundamental nesta etapa da vida da mulher, pois visa restabelecer a atividade funcional do membro acometido, além de significar um conjunto de possibilidades terapêuticas físicas passíveis de intervir e diminuir o tempo de recuperação, com retorno mais rápido às atividades cotidianas e ocupacionais. A atuação multiprofissional para a reabilitação do membro superior deve começar, o mais precocemente possível, colaborando com a reintegração social da mulher, contribuindo com a recuperação da funcionalidade e da melhoria da qualidade de vida das pacientes ${ }^{10}$.

Diante do exposto, o objetivo deste estudo foi avaliar o efeito agudo da abordagem indireta no tecido conjuntivo sobre a dor e a propriocepção do membro superior de mulheres pós-mastectomia radical modificada unilateral.

\section{MATERIAL E MÉTODO}

Estudo quase experimental, com abordagem quantitativa, com pré e pós-teste, sem grupo-controle, 
aprovado pelo Comitê de Ética em Pesquisa (CEP) da Universidade Federal de Santa Maria (UFSM), RS, sob o protocolo número 512.084. Previamente ao início da coleta de dados, as voluntárias assinaram o Termo de Consentimento Livre e Esclarecido (TCLE).

A população da pesquisa foi mulheres submetidas à mastectomia radical modificada unilateral, com faixa etária entre 35 e 59 anos, sendo que a amostra, por conveniência, constituiu-se de 20 mulheres que aceitaram participar do estudo. Entretanto, 08 voluntárias foram excluídas por não apresentarem dor no momento do pré-teste, totalizando uma amostra de 12 mulheres. Além da dor, constituíram critérios de exclusão: mulheres que realizaram outro tipo de cirurgia de mama, que não a mastectomia radical modificada; aquelas que estavam em tratamento de radio ou quimioterapia; dificuldade de compreensão na realização do teste e na resposta ao instrumento de pesquisa. Também foram excluídas mulheres com comprometimento severo da amplitude de movimento articular que inviabilizou a aplicação do instrumento de coleta de dados (cinesiômetro).

A amostra foi proveniente do Ambulatório de Fisioterapia do Hospital Universitário de Santa Maria, centro do estado do Rio Grande do Sul. As participantes foram encaminhadas para o Centro de Referência à Saúde do Trabalhador (CEREST), por maior disponibilidade, onde foram avaliadas. O CEREST- Região Centro consiste em um serviço de referência à saúde do trabalhador para a $4^{\mathrm{a}}$ Coordenadoria Regional de Saúde, sendo que esta, por sua vez, abarca 31 municípios da região. Neste local desenvolvem-se atividades dos cursos de graduação e pósgraduação da UFSM, constituindo-se em um espaço de integração ensino-serviço. Especificamente, neste local são realizados atendimentos às mulheres em tratamento para o câncer de mama.

A coleta de dados se deu no decorrer do ano de 2015, por meio do preenchimento do questionário sociodemográfico, elaborado pelas pesquisadoras, com o objetivo de caracterizar a amostra quanto à faixa etária, índice de massa corporal (IMC), situação conjugal, escolaridade e dados reprodutivos. Posteriormente, aplicou-se o Disabilities of the Arm, Shoulder and Hand $(\mathrm{DASH})^{11}$ para a avaliação da funcionalidade do membro superior. Esse instrumento contou com 30 questões autoaplicáveis e dois módulos opcionais, que avaliaram os sintomas e as funções físicas, sociais e psicológicas e foi desenvolvido para ser utilizado em pacientes que apresentavam qualquer distúrbio, em qualquer articulação do membro superior. Este estudo utilizou as 30 primeiras questões, mais o módulo opcional referente às atividades de trabalho (incluindo tarefas domésticas). Utilizou- se, ainda, a Escala Visual Analógica da Dor (EVA), que consiste de uma linha reta numerada de zero a dez, nas quais zero significa ausência de dor e dez, a pior dor imaginável, que foi utilizada para avaliar a intensidade da dor das participantes no momento da coleta.

Para avaliar a propriocepção, foi utilizado o cinesiômetro (Figura 1), conforme o protocolo de Paixão ${ }^{6,7,12}$, que é composto de uma base de madeira onde estão afixados os graus de $0^{\circ}$ a $180^{\circ}$ para determinar os ângulos, e um braço móvel no qual a voluntária era orientada a mover em movimentos de rotação interna e externa do ombro até determinado ângulo para a avaliação da propriocepção. A avaliação da propriocepção foi realizada com a paciente em sedestação, à frente de uma mesa na qual repousava o instrumento, de modo a ficar confortavelmente acomodada. $\mathrm{O}$ tronco da paciente permanecia apoiado na cadeira e o membro superior homolateral à mastectomia na posição supinada era colocado sobre o equipamento repousado sobre uma mesa. Em seguida, a paciente tinha seus olhos vendados de modo a evitar qualquer percepção visual do movimento a ser executado. Nesse momento, os pesquisadores demonstravam as três posições em que o movimento deveria ser realizado, sendo estes em posições prédeterminadas: $90^{\circ}, 45^{\circ}$ e $105^{\circ}$ graus, respectivamente.

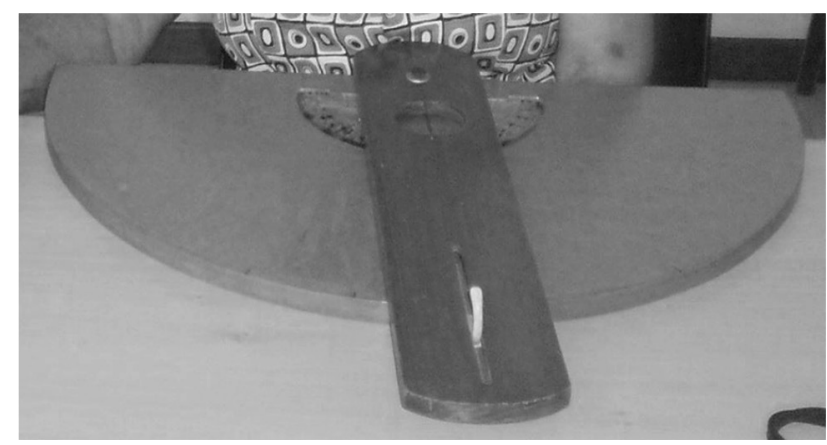

Figura 1 - Cinesiômetro, aparelho utilizado para avaliar a propriocepção de membros superiores, segundo o protocolo de Paixão (Foto do arquivo pessoal da pesquisadora)

Em cada ângulo demonstrado, o examinador mantinha o membro superior da paciente, por cerca de 10 segundos, para que esta percebesse o posicionamento do seu membro; repetia-se o mesmo procedimento nas 3 angulações descritas e retomava-se à posição inicial $\left(0^{\circ}\right.$ grau $)$. Neste momento, iniciava-se o teste de modo ativo em que a paciente conduzia o braço móvel do equipamento até os ângulos que ela acreditava que representassem as angulações determinadas pelo equipamento, demonstradas 
anteriormente $\left(90^{\circ}, 45^{\circ}\right.$ e $105^{\circ}$ graus). $\mathrm{O}$ resultado era o erro, ou seja, quantos graus faltaram ou excederam o ponto exato do ângulo determinado. A avaliação da propriocepção foi realizada a partir da média de três repetições do movimento, de modo cego, ou seja, o questionário foi aplicado por um pesquisador e o cinesiômetro por outro, a fim de minimizar vieses de avaliação.

Após a avaliação, as pacientes foram submetidas à abordagem indireta no tecido conjuntivo, pois essas técnicas enfocam a rede miofascial como um todo. Entende-se por rede miofascial aquela formada pelo tecido conjuntivo, o qual se constitui como uma unidade que se estende por todo corpo, sendo que qualquer movimento, manobra aplicada em um segmento produz efeitos a distância ${ }^{8}$.

A massagem miofascial indireta consiste na liberação da miofáscia por meio de movimentos suaves do tecido conjuntivo. São movimentos de balanceio, agitação ou tração suave do segmento trabalhado. Foram utilizadas três técnicas: Roncking, Jostling e Flopping ${ }^{8}$. A manobra de Rocking envolve movimentos passivos de balanceio, laterais ou circulares, ou combinações entre estes, com o membro para cima e para baixo, por intermédio da vibração produzida pelo terapeuta e transmitida a todo o corpo do paciente. O Jostling consiste em um tipo de vibração produzida pelo movimento rítmico, rápido e continuado gerado pelo terapeuta, que é transmitido a todo o corpo do paciente, como se fossem sacudidelas. O Flopping, por sua vez, consiste em oscilações, agitações produzidas em sentido latero-lateral em que o membro do paciente é movido de forma controlada sobre a mesa ${ }^{8}$.

O conjunto das três manobras foi aplicado durante, aproximadamente 15 minutos, em uma intervenção com avaliação pré e pós-massagem miofascial, sendo que a mulher foi colocada na posição de decúbito dorsal sobre a maca com o corpo em completa extensão e a cabeça apoiada sobre o travesseiro.

Para a análise estatística foi utilizado o teste de normalidade Shapiro-Wilk. Os dados considerados assimétricos foram transformados, logaritimicamente, objetivando a simetria, possibilitando a utilização de testes estatísticos mais robustos.

Para a análise pré e pós dos dados simétricos, foi utilizado o Teste T Pareado. Já, para a comparação de dados assimétricos, foi utilizado o teste Wilcoxon. O nível de significância adotado foi de $\mathrm{p} \leq 0,05$.

\section{RESULTADOS}

As mulheres participantes do estudo eram da cor branca, com média de idade de 55,83 $( \pm 9,34)$ anos, tendo como IMC médio $25,72( \pm 2,52) \mathrm{kg} / \mathrm{cm}^{2}$. A Tabela 1 apresenta a caracterização da amostra quanto à situação conjugal, escolaridade e dados reprodutivos como: número de gestações, idade da primeira gestação, idade da menarca, idade da menopausa e funcionalidade do membro superior de mulheres submetidas à mastectomia radical modificada unilateral.

Tabela 1 - Situação conjugal, escolaridade, dados reprodutivos e funcionalidade do ombro de mulheres submetidas à mastectomia radical modificada unilateral

\begin{tabular}{|c|c|c|}
\hline Variáveis & \multicolumn{2}{|c|}{ n (\%) } \\
\hline SITUAÇÃO CONJUGAL & \multirow{4}{*}{\multicolumn{2}{|c|}{$\begin{array}{c}1(8,33) \\
10(83,33) \\
1(8,33)\end{array}$}} \\
\hline Solteira & & \\
\hline Casada & & \\
\hline Separada & & \\
\hline ESCOLARIDADE & \multirow{5}{*}{\multicolumn{2}{|c|}{$\begin{array}{c}3(25) \\
1(8,33) \\
6(50) \\
2(16,66)\end{array}$}} \\
\hline Ensino Fund. Incompleto & & \\
\hline Ensino Médio Incompleto & & \\
\hline Ensino Médio Completo & & \\
\hline Ensino Superior Completo & & \\
\hline Variáveis & Média & DP \\
\hline \multicolumn{3}{|l|}{ DADOS REPRODUTIVOS } \\
\hline $\mathrm{N}^{\mathrm{o}}$ de gestações & 2,66 & $\pm 1,15$ \\
\hline Idade da $1^{\mathrm{a}}$ gestação & 22,33 & $\pm 3,47$ \\
\hline Idade da menarca & 11,91 & $\pm 1,16$ \\
\hline Idade da menopausa & 47,83 & $\pm 5,49$ \\
\hline \multicolumn{3}{|l|}{ FUNCIONALIDADE } \\
\hline DASH1 & 22,15 & $\pm 14,99$ \\
\hline DASH3 & 30,18 & $\pm 21,77$ \\
\hline Total do DASH & 26,16 & $\pm 17,28$ \\
\hline
\end{tabular}

Valores expressos em n, média e desvio-padrão (dp).

O tempo médio de pós-cirúrgico foi de 28,08 ( \pm $24,90)$ meses. Todas as mulheres realizaram um ou mais tratamentos adjuvantes, sendo estes, a quimioterapia, a radioterapia e a hormonioterapia. Todas relataram sinais de menopausa, após começar o tratamento complementar do câncer de mama.

O lado predominante da cirurgia de mastectomia foi o lado direito, com 9 (75\%) casos, assim como o membro superior dominante destas mulheres, também, foi o direito, ou seja, a maioria das pacientes era destras. Quanto à reabilitação, as mulheres referiram que realizaram fisioterapia, com média de tempo de 7,58 $( \pm 10,33)$ meses, sendo que 2 mulheres não haviam realizado nenhum tratamento de reabilitação até o momento da avaliação.

A avaliação da propriocepção, por meio do cinesiômetro, no pré-teste, apresentou média de erro de $14,69^{\circ}$, sendo que no pós-teste foi de $11,38^{\circ}$. Na avaliação 
da dor pela EVA identificou-se que, no pré-teste, a média foi de 3,33, enquanto que no pós-teste, foi de 2,66 (Tabela 2). Entretanto, apesar da redução das médias, não houve resultado estatisticamente significativo.

Tabela 2 - Avaliação da dor e propriocepção pré e pós-manobra miofascial indireta

\begin{tabular}{l|c|c|c}
\hline Variáveis & Pré & Pós & P \\
\hline Propriocepção & $14,69^{\circ}( \pm 6,89)$ & $11,38^{\circ}( \pm 8,00)$ & 0,258 \\
Dor & $3,33( \pm 2,60)$ & $2,66( \pm 2,30)$ & 0,072 \\
\hline
\end{tabular}

Valores expressos em média e desvio-padrão (dp).

\section{DISCUSSÃO}

As participantes do estudo eram mulheres brancas (100\%), sendo $83,33 \%$ casadas, com média de idade de 55,83 anos $( \pm 9,34)$. A média do IMC das participantes foi de $25,72( \pm 2,52) \mathrm{kg} / \mathrm{cm}^{2}$, o que pode ser considerado sobrepeso. De acordo com o Instituto Nacional do Câncer, a obesidade e o sobrepeso são considerados fatores comportamentais e incidem como fator de risco para o desenvolvimento do câncer de mama $^{13}$. Em relação à escolaridade, 6 das participantes $(50 \%)$ possuíam ensino médio completo e $3(25 \%)$, ensino fundamental incompleto.

Quanto aos dados reprodutivos das mulheres participantes desta pesquisa, a idade média para a menarca foi de $11,91( \pm 1,16)$ anos, e para a menopausa, 47,83 $( \pm 5,49)$ anos.

Ainda, como dados reprodutivos, a idade média da primeira gestação foi de 22,33 $( \pm 3,47)$ anos, enquanto que a média de filhos foi de 2,66 $( \pm 1,15)$. Com relação à idade da primeira gestação, constatou-se que $94,4 \%$ das mulheres deste estudo tiveram o primeiro parto até os 30 anos de idade. Sobre o tratamento adjuvante como radioterapia, quimioterapia e hormonioterapia, todas realizaram um ou mais tratamentos.

Em relação ao tempo pós-cirurgia para o tratamento do câncer de mama, a média foi de 28,08 $( \pm 26,90)$ meses, e o lado predominante da cirurgia foi o lado direito, com 9 (75\%) casos. Em um estudo realizado com 121 mulheres, que realizaram cirurgia para a retirada do câncer, o tempo pós-cirurgia foi de até 24 meses, totalizando $67,7 \%$ da amostra; $33,3 \%$ mais que 24 meses e o lado acometido foi o direito, com $52,1 \%{ }^{14}$. O tempo de tratamento fisioterapêutico realizado pelas participantes desta pesquisa foi de 7,58 ( $\pm 10,33)$ meses, portanto, menos tempo, quando comparado ao estudo citado.

Apesar de a literatura atual não apontar um instrumento para avaliar a funcionalidade na população com câncer de mama ${ }^{15}$, o DASH tem sido frequentemente utilizado como medida funcional para avaliar desordens no membro superior decorrentes da abordagem terapêutica ${ }^{16}$.

Neste estudo, a média da funcionalidade (pelo $\mathrm{DASH}$, as 30 primeiras questões e o módulo opcional referente às atividades do trabalho) foi de $26,16( \pm 17,28)$. Em um estudo similar que avaliou a funcionalidade pelo DASH em mulheres com câncer de mama em Juiz de Fora, (MG), foi encontrada, como média da funcionalidade, $21,09( \pm 19,56)$, sugerindo melhora na função do membro superior ${ }^{14}$. Segundo Cheng et al. ${ }^{17}$ e Orfale et al. ${ }^{11}$, o escore alto (100) indica disfunção severa, e um escore baixo (0), sem disfunção. Os dados do DASH não foram estatisticamente significativos, mas as participantes, segundo esses autores, obtiveram um escore baixo, ou seja, pouco comprometimento funcional do membro superior.

Barbosa $^{14}$ relata que a funcionalidade do membro superior avaliado, por meio do DASH, demostrou que $34,7 \%$ dessa funcionalidade dependeram da variável da dor. Neste estudo, a dor, também, foi avaliada, antes e depois, da abordagem indireta no tecido conjuntivo, em que a dor pré-tratamento apresentou média de 3,33 $( \pm 2,60)$ e pós-tratamento, $2,66( \pm 2,30)$, na EVA. Porém, não foi estatisticamente significativa essa redução e nem se obteve correlação com nenhuma das demais variáveis investigadas. A intensidade da dor nas mulheres avaliadas nesse estudo foi leve, pois a maioria havia realizado cirurgia há mais de um ano e, ao mesmo tempo, tratamento fisioterapêutico.

Outro motivo pode ser decorrente da aplicação da técnica em um único momento, pois a fáscia é o componente básico do sistema musculoesquelético do corpo e, em circunstâncias normais, ela deve ser flexível e deslizante. No entanto, em casos de traumatismos, processos inflamatórios, cirurgias, estresse, criam-se restrições e aderências na fáscia, o que faz com que ela se torne mais sólida e, dessa forma, encurte suas fibras, o que cria pressão em áreas sensíveis, provocando dor e restrições de movimento ${ }^{9}$, carecendo de tratamentos longitudinais.

Os movimentos indiretos no tecido conjuntivo se concentram em trazer conforto às áreas "presas", pois as manobras realizadas, Jostling, Roncking e o Flopping, podem causar relaxamento muscular ${ }^{8}$, consequentemente, o alívio da dor. Isto, também, pode ter influenciado na avaliação da propriocepção, visto que com o membro superior mais relaxado e com menos dor, as participantes conseguiram ter uma percepção melhor do seu corpo, da posição do seu membro superior no espaço, sendo, possivelmente, um indicativo da melhora da dor e da propriocepção pós-massagem miofascial. 
Em um estudo ${ }^{18}$ realizado em Presidente Prudente$\mathrm{SP}$, que avaliou o efeito da terapia manual (as manobras não foram especificadas pelos autores do estudo) em pacientes mastectomizadas com dor nos músculos da cintura escapular e cervical, houve uma diminuição da dor em todas as 19 pacientes avaliadas. No estudo supracitado, a intensidade média da dor declarada no início da sessão foi de 6,6 (escala analógica da dor, de 0 a 10), e no final desta primeira sessão foi reduzida para 4,6. As pacientes declararam que a intensidade da dor diminuiu em torno de 2 pontos em relação à dor que sentiam antes do tratamento. Ao longo das sessões, houve diminuição da dor e a intensidade média declarada como 3,4 e, no final da última sessão, como 1,37. No estudo citado, os autores concluíram que a terapia manual foi efetiva para a redução da dor miofascial em mastectomizadas, contribuindo para a melhora da qualidade de vida dessas mulheres ${ }^{18}$.

A avaliação da propriocepção apresentou como resultado uma média de erro absoluto, pré-manobra, de $14,69^{\circ}( \pm 6,89)$ e pós-manobra, de $11,38^{\circ}( \pm 8,00)$. Pode-se inferir que quanto menor o erro absoluto, melhor é o desempenho proprioceptivo do indivíduo, consequentemente, há melhora da propriocepção ${ }^{6}$. Esta é fundamental para a execução de movimentos e uma importante fonte de informação sensorial, que dá ao indivíduo noção sobre as posições e sensações. Estatisticamente, as diferenças nas angulações do erro absoluto não foram significativas, porém, os resultados expressos nas médias revelaram diferenças entre pré e pósmanobra, mostrando que houve melhora na propriocepção das participantes após a manobra da abordagem indireta no tecido conjuntivo.

Nessesentido,váriosgrupos demecanoceptores podem ser estimulados, ao máximo, para aumentar a propriocepção de diferentes estruturas musculoesqueléticas ${ }^{19,20}$. Os mecanoceptores cutâneos podem ser estimulados por meio

\section{REFERÊNCIAS}

1. Instituto Nacional do Câncer (INCA). Estimativa 2012: incidência de câncer no Brasil [citado 15 jun. 2015]. Disponível em: http://www.inca.gov.br/estimativa/2012/ index.asp? ID $=2$.

2. Silva SED, Vasconcelos EV, Santana ME, Rodrigues ILA, Leite TV, Santos LMS, et al. Representações sociais de mulheres mastectomizadas e suas implicações para o de eventos dinâmicos na pele, como massagem, friç̧ão ou vibração e por técnicas de movimentos rítmicos ou oscilatórios $^{20}$. Isso corrobora que a abordagem indireta do tecido conjuntivo realizada nas mulheres nessa pesquisa estimulou os mecanoceptores, pois as participantes melhoraram a execução do movimento e a informação sensorial, que é a que dá ao indivíduo a noção sobre as posições e as sensações, ou seja, melhorou a propriocepção, pós-manobra.

Um estudo que analisou a propriocepção dos membros superiores de 8 mulheres praticantes da habilidade motora específica de natação no processo de familiarização ao meio líquido ${ }^{6}$, também, não encontrou diferença estatisticamente significativa, corroborando com os resultados da presente pesquisa. O presente estudo apresentou médias inferiores de propriocepção de membro superior em relação ao estudo citado, o que denotou o baixo nível de propriocepção dos sujeitos aqui analisados.

Alguns resultados semelhantes foram encontrados em outro estudo, no qual foi verificada a propriocepção de membros superiores de idosas praticantes de hidroginástica, em que a média foi de $11,30^{\circ}$ no Grupo 1 (59-69 anos), e 12,95 , no Grupo 2 (70-84 anos). Este estudo citado ressalta, ainda, que quanto mais avançada é a idade, maior é a perda motora, e, consequentemente, a propriocepção ${ }^{21}$.

\section{CONCLUSÃO}

Não houve diferença significativa pré e pósabordagem indireta no tecido conjuntivo sobre a dor e a propriocepção em mulheres submetidas à mastectomia radical modificada. Isso pode ter ocorrido pelo pequeno número amostral, pelo curto tempo da realização da manobra ou pela aplicação única da técnica. Também, não foram encontrados na literatura, até o momento, trabalhos semelhantes que permitissem comparação com os achados deste estudo.

autocuidado. Rev Bras Enf. 2010;5(6):727-34. http://dx.doi. org/10.1590/S0034-71672010000500006.

3. Barbosa JAN, Amorin MHC, Zandonade E, Delaprane ML. Avaliação da postura corporal em mulheres com câncer de mama. Rev Bras Ginecol Obstet. 2013;35(5):215-20. Disponível em: http://www.scielo.br/pdf/rbgo/v35n5/05.pdf. 
4. Brasil. Ministério da Saúde. Secretaria de Atenção à Saúde. Protocolos clínicos e diretrizes terapêuticas em oncologia. Brasília; 2014 [citado 19 jan. 2017]. Disponível em: http:// bvsms.saude.gov.br/bvs/publicacoes/protocolos_clinicos diretrizes_terapeuticas_oncologia.pdf.

5. Cezar K, Nascimento APC. Qualidade de vida de pacientes pós-mastectomizadas em reabilitação oncológica. J Health Sc. 2014;16(1):29-32. http://dx.doi.org/10.17921/24478938.2014v16n1p\%25p.

6. Antes DL, Katzer JI, Corazza ST. Coordenação motora fina e propriocepção de idosas praticantes de hidroginástica. RBCEH, 2008;5(2):24-32. http://dx.doi.org/10.5335/rbceh.2012.109.

7. Deshpande N, Connelly DM, Culham EG, Costigan PA. Reliability and validity of ankle proprioceptive measures. Arch Phys Med Rehabil. 2003;84:883-9. http://dx.doi.org/10.1016/ S0003-9993(03)00016-9

8. Dixon MW. Massagem miofascial. fisioterapia práticaRio de Janeiro: Guanabara-Koogan; 2007. (Série Physio).

9. Myers TW. Trilhos Anatômicos: meridianos miofasciais para terapeutas manuais e do movimento. 3a ed. São Paulo: Manole; 2017.

10. Giacon FP, Peixoto BO, Kamonesky DH, Sampaio Neto, LF. Efeitos do tratamento fisioterapêutico no pós-operatório de câncer de mama na força muscular e amplitude de movimento de ombro. J Health Sci Inst. 2013;31(3):316-9. Disponível em: https://www. unip.br/comunicacao/publicacoes/ics/edicoes/2013/03_jul-set/ V31_n3_2013_p316a319.pdf.

11. Orfale AG, Araujo PMP, Ferraz MB, Natour J. Translation into Brazilian Portuguese, Cultural Adaptation and Evaluation of the Reliability of the Disabilities of the Arm, Shoulder and Hand Questionnaire. Braz J Med Biol Res. 2005;38(2):293302. http://dx.doi.org/10.1590/S0100-879X2005000200018.

12. Paixão JS. Efeitos do plano motor na aquisição, retenção e transferência de uma destreza motora fechada [Dissertação]. Santa Maria: Universidade Federal de Santa Maria; 1981.

13. Instituto Nacional de Câncer José Alencar Gomes da Silva (INCA). Coordenação Geral de Ações Estratégicas. Coordenação de Prevenção e Vigilância. Estimativa 2016: incidência de câncer no Brasil. Rio de Janeiro; 2015 [citado 19 jan. 2017]. Disponível em: http://www2.inca.gov.br/ wps/wcm/connect/acoes_programas/site/home/nobrasil/ programa_controle_cancer_mama/fatores_risco.

14. Barbosa PA. Avaliação da qualidade de vida e impacto funcional em mulheres com câncer de mama pósintervenção cirúrgica na cidade de Juiz de Fora, Minas Gerais [Dissertação]. Juiz de Fora: Universidade Federal de Juiz de Fora; 2014. Disponível em: http://www.ufjf.br/pgsaudecoletiva/files/2014/02/ Disserta\%C3\%A7\%C3\%A3o-Priscila-Almeida-Barbosa. pdf.

15. Carvalho FN, Koifman RJ, Bergmann A. Classificação Internacional de Funcionalidade, Incapacidade e Saúde em Mulheres com Câncer de Mama: proposta de instrumentos para mensuração. Cad Saúde Pública (Rio de Janeiro). 2013;29(6):1083-93. http://dx.doi.org/10.1590/S0102$311 X 2013000600005$.

16. Institute for Work and Health. The DASH and Quick DASH disabilities of the arm, shoulder, \& hand. Toronto; 2012. (The DASH and Quick DASH e-bulletin) [cited 2017 Jan 18]. Available from: http://www.dash.iwh.on.ca/sites/dash/files/ dash_e-bulletin_2013_summer.pdf.

17. Cheng HMS. Disabilities of the Arm, Shoulder, and Hand DASH: análise da estrutura fatorial da versão adaptada para o português [Dissertação]. Belo Horizonte: Escola de Educação Física, Fisioterapia e Terapia Ocupacional, Universidade Federal de Minas Gerais; 2006. Disponível em: http://www. eeffto.ufmg.br/eeffto/DATA/defesas/20150825160626.pdf.

18. Pancioni GC, Carmo EM, Pereira RS, Gomes PRL. Efeito da terapia manual em pacientes mastectomizadas com dor nos músculos da cintura escapular e cervical. Ter Man. 2010;38(8):305-13.

19. Lederman E. Fundamentos da terapia manual. Fisiologia, neurologia e psicologia. São Paulo: Manole; 2001.

20. Drews R, Cardozo PL, Corazza ST, Flôres FS. Análise do desempenho motor de escolares praticantes de futsal e voleibol. Motricidade. 2013;9(3):105-16. http://dx.doi. org/10.6063/motricidade.9(3).1123.

21. Corazza ST, Pereira FÉ, Villis JMC. Propriocepção e a familiarização no meio líquido. Rev Digital (Buenos Aires). 2005;10(82) [citado 18 jan. 2017]. Disponível em: http:// www.efdeportes.com/efd82/propio.htm.

Recebido em: 21.01.16

Aceito em: 24.01.17 